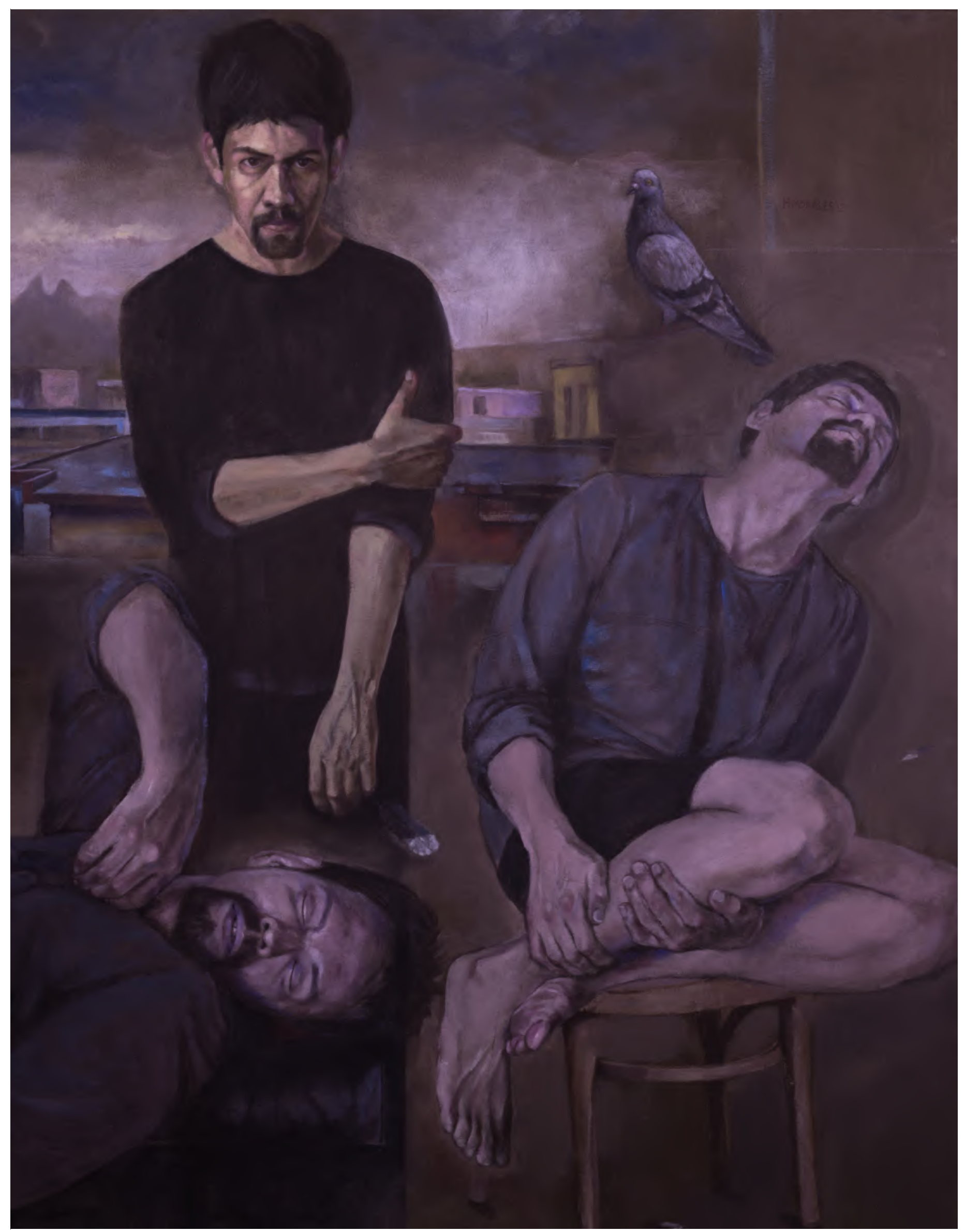

ESTUDIO PARA TRES RETRATOS | Temple sobre tela y madera $\mid 0.70 \times 100 \mathrm{cms}$ 


\section{PROYECTO DE INNOVACIÓN PARA LA ASIGNATURA DE PARA ESCOLARES}

\section{INNOVATION PROJECT FOR THE SUBJECT OF SCHOOLCHILDREN}

\section{RESUMEN}

Durante el ciclo escolar Semestre A 2020 se presentaron situaciones de orden internacional que atentan hasta hoy en día con la salud, es el caso, del SARS-COV 2 que surgió en China en diciembre de 2019, provocando la enfermedad llamada COVID-19, que se extendió por el mundo y fue declarada pandemia por la Organización Mundial de la Salud. Esto trajo como consecuencia un nuevo paradigma educativo, donde el salón de clases se convirtió en un dispositivo móvil, el docente como un facilitador electrónico y el alumno en un internauta educativo. El presente trabajo propone la incorporación del aprendizaje móvil o m-Learning con el uso de las plataformas digitales TED y ZOOM dentro del Colegio de Ciencias y Humanidades de la Universidad Juárez del Estado de Durango.
ABSTRACT

During the Semester A 2020 school year, there were international situations that threaten health to this day, it is the case of SARS-COV 2 that emerged in China in December 2019, causing the disease called COVID-19, which it spread throughout the world and was declared a pandemic by the World Health Organization. This resulted in a new educational paradigm, where the classroom became a mobile device, the teacher as an electronic facilitator and the student an educational Internet user. This work proposes the incorporation of mobile learning with the use of digital platforms TED and Z00M within the College of Sciences and Humanities of the Universidad Juarez del Estado de Durango. Covid-19, Educational, Virtual. 
a pandemia global provocada por

el Covid-19 trajo consigo la inmovilización de la población mundial en los sectores económicos, sociales, culturales y educativos; donde las clases fueron suspendidas de forma presencial para dar paso a la educación a distancia en su formato virtual. Los participantes de la enseñanza (instituciones, directivos, docentes) han tenido que buscar nuevas herramientas virtuales que les permitan establecer una comunicación clara y directa con el alumno, investigando nuevas formas de enseñanza procurando mantener la calidad educativa de lo que es la educación presencial.

Los alumnos a su vez, como nativos digitales, se han convertido en autodidactas, pues buscan más información sobre el tema expuesto por el docente que pueda cumplir con sus expectativas y cuestionamientos, dando así paso a la necesidad de una innovación educativa para solucionar la inquietud estudiantil de aprendizaje por medios electrónicos.

El presente trabajo propone la incorporación del aprendizaje móvil o mobile learning 0 m-learning con el uso de las plataformas digitales TED y ZOOM para la clase de Para-escolares 3A, 3B, 3C, 4A y $4 \mathrm{~B}$ dentro del Colegio de Ciencias y Humanidades de la Universidad Juárez del Estado de Durango.

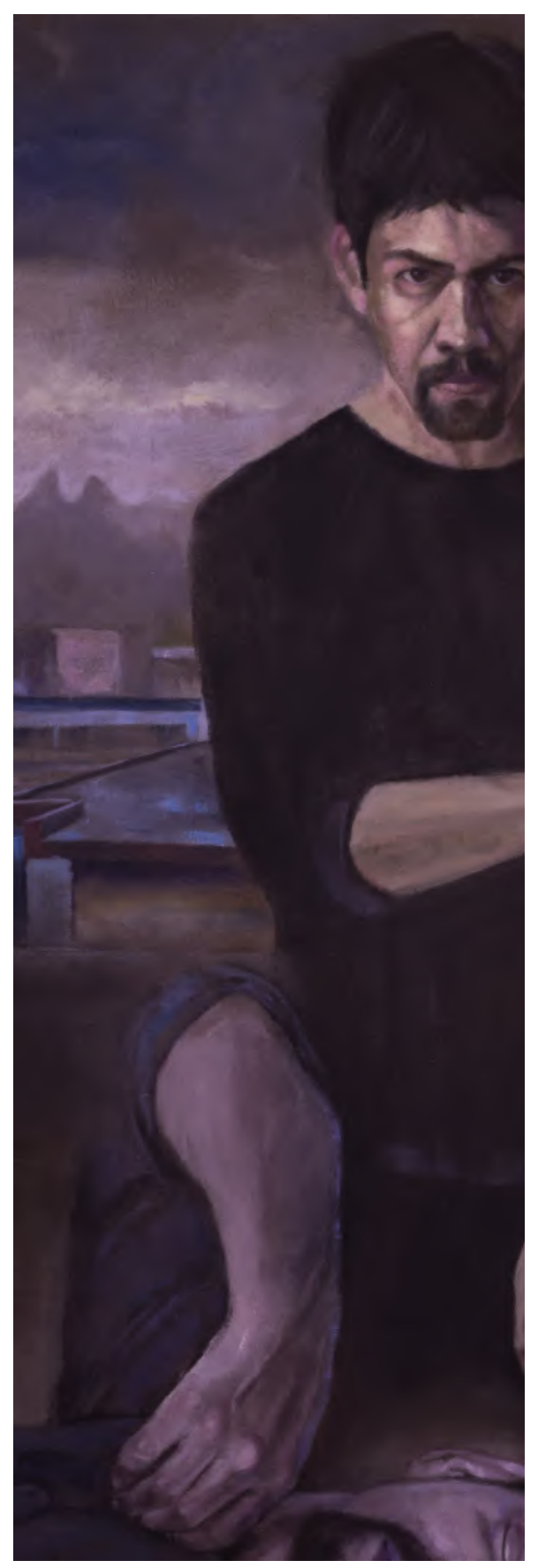

Por este método se pretende dar a los alumnos herramientas dentro del campo disciplinar de las humanidades en los 2 semestres de la materia mencionada, para desarrollar las habilidades socioemocionales, la orientación educativa, la orientación 1 CCH vocacional, promoción del crecimiento personal, entre otros temas de carácter formativo.

Dicho proyecto nace por la necesidad de clases a distancia, pero su intención es perfeccionarlo y que se pueda adoptar como una herramienta didáctica más dentro de las clases presenciales, pues puede aportar beneficios no sólo a los adolescentes sino también a la institución, quienes tendrían mayor gama de conferencistas en temas y culturas, disponibles $24 / 7$ y de forma gratuita.

Un proyecto es el conjunto de actividades que se realizan en un lugar, tiempo, con recursos determinados y así alcanzar los objetivos deseados, que den solución a un problema (Olmedo, 2020).

En los principios de los años 70, podemos encontrar la innovación educativa con Huberman, Fullen y Pomffrett y Harvelock. La innovación educativa es un proceso que involucra la selección, organización y utilización creativa de elementos vinculados a la gestión institucional, el currículum y/o la enseñanza,

\section{Un proyecto es el conjunto de actividades que se realizan en un lugar, tiempo, con recursos determinados y así alcanzar los objetivos deseados, que den solución a un problema (Olmedo, 2020).}




\section{El lema es: Ideas Que Merece La Pena Difundir. En esta comunidad mundial se reciben a personas de todas las disciplinas y culturas que buscan una comprensión más profunda del mundo (TED, 2020).}

siendo normal que una innovación educativa impacte más de un ámbito, ya que suele responder a una necesidad o problema que regularmente requiere una respuesta integral (Cárdenas, 2013).

Este trabajo expone un proyecto de innovación para la asignatura de paraescolares que busca subsanar la falta de conferencias que aborden los temas relativos a la adolescencia como lo son relaciones con sus pares, su entorno familiar y social, toma de decisiones, autoestima, identificación de vocación, emprendimiento, debido al confinamiento por Covid-19 y en un futuro como apoyo a las conferencias presenciales que se retoman cuando las circunstancias pedagógicas lo permitan.

Así el alumno no considera el aprendizaje de estos temas de manera memorística o completamente teórica, sino que escuchara el tema de personas de su edad famosos, personas que han pasado por temas con los que se sienten identificados y con un vocabulario más coloquial y del dominio del adolescente.

Esto se logra por medio de la plataforma TED Talks, que combina el arte de la oratoria con las tecnologías audiovisuales y de la comunicación y las experiencias personales de gente común hasta personas destacadas en diferentes ámbitos.

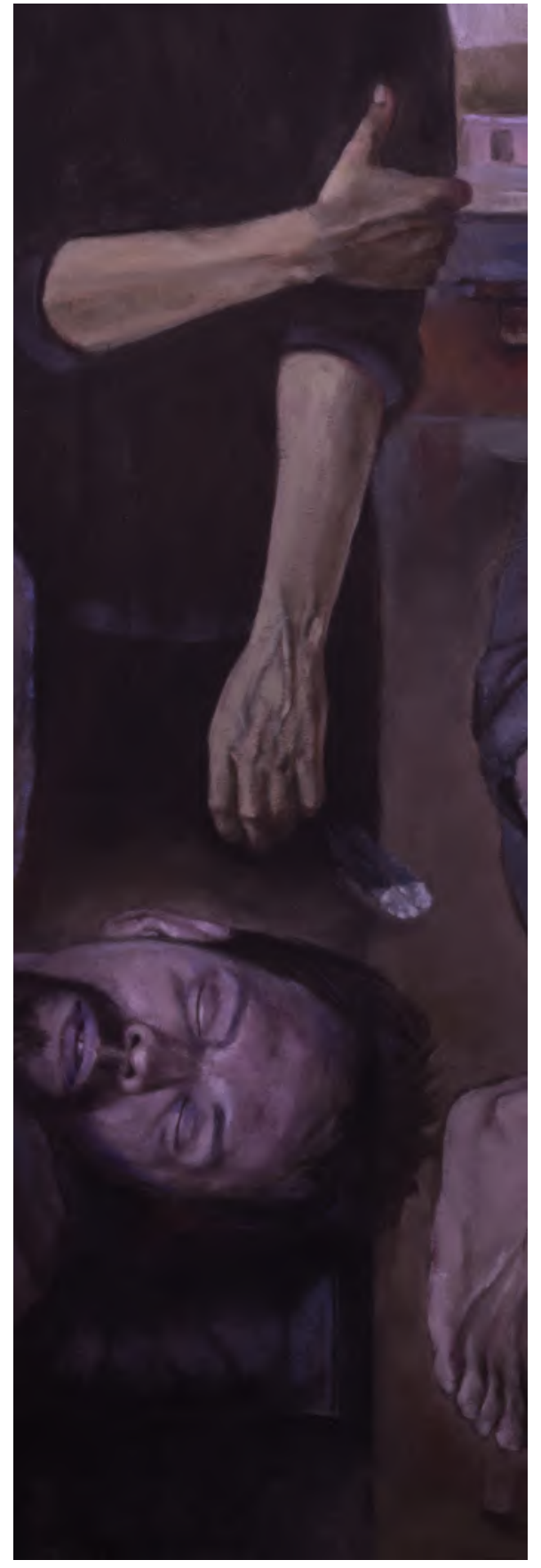

TED es una organización estadounidense no gubernamental y sin ánimo de lucro cuya función es la difusión de ideas a nivel mundial a través de los congresos anuales conocidos como TED Conference o TED Talks. El lema es: Ideas Que Merece La Pena Difundir. En esta comunidad mundial se reciben a personas de todas las disciplinas y culturas que buscan una comprensión más profunda del mundo (TED, 2020). El docente a cargo de la asignación buscará la conferencia más acercada al tema que se quiera tratar en clase, esto como apoyo al material teórico de la misma, para pedir a los alumnos que formen un criterio sobre el tema y poder debatir en una conferencia virtual con los otros participantes de la clase por medio de la plataforma llamada ZOOM.

Con el servicio de la plataforma Z00M se pueden realizar videoconferencias en un formato que permite guardar el trabajo dentro de la nube, o bien, en el ordenador que utiliza para realizar la reunión. Además, permite el chat con las personas presentes en la conferencia de manera general 0 en privado con algún integrante. Esto permite la posibilidad que sí algún alumno no puede estar presente dentro de la clase-conferencia, pueda verlo más tarde y dar su punto de vista 0 reflexión por otro medio que el mismo docente condicione para ello. 


\section{Tabla 1. Competencias}

\begin{tabular}{l|l|l|}
\multicolumn{1}{|c|}{ COMPETENCIA GENÉRICA } & \multicolumn{1}{|c|}{ ATRIBUTO } \\
\hline \multicolumn{2}{|c|}{ SE AUTODETERMINA Y CUIDA DE SÍ } \\
\hline $\begin{array}{l}\text { Se autodetermina y cuida de sí. } \\
\text { ne conoce y valora a sí mismo y aborda problemas y retos te- }\end{array}$ & $\begin{array}{l}\text { Elige alternativas y cursos de acción con base en criterios sus- } \\
\text { tentados y en el marco de un proyecto de vida. Analiza críti- } \\
\text { camente los factores que influyen en su toma de decisiones. }\end{array}$ \\
\hline Elige y practica estilos de vida saludables. & $\begin{array}{l}\text { Toma decisiones a partir de la valoración de las consecuencias } \\
\text { de distintos hábitos de consumo y conductas de riesgo. } \\
\text { Cultiva relaciones interpersonales que contribuyen a su desa- } \\
\text { rrollo humano y el de quienes lo rodean. }\end{array}$ \\
\hline
\end{tabular}

\section{SE EXPRESA Y COMUNICA}

Escucha, interpreta y emite mensajes pertinentes en distintos contextos mediante la utilización de medios, códigos y herramientas apropiados.
Expresa las ideas y conceptos mediante representaciones lingüísticas, matemáticas o gráficas.

Identifica las ideas clave en un texto o discurso oral e infiere conclusiones a partir de ellas.

Maneja las tecnologías de la información y la comunicación para obtener información y expresar ideas.

\section{PIENSA CRÍTICA Y REFLEXIVAMENTE}

\begin{tabular}{l|l}
\hline $\begin{array}{ll}\text { Desarrolla innovaciones y propone soluciones a problemas a } \\
\text { partir de métodos establecidos. }\end{array}$ & $\begin{array}{l}\text { Sigue instrucciones y procedimientos de manera reflexiva, } \\
\text { comprendiendo como cada uno de sus pasos contribuye al al- } \\
\text { cance de un objetivo } \\
\text { Utiliza las tecnologías de la información y comunicación para } \\
\text { procesar e interpretar información. }\end{array}$ \\
\hline
\end{tabular}

\section{APRENDE DE FORMA AUTÓNOMA}

Aprende por iniciativa e interés propio a lo largo de la vida.

\section{de conocimiento.}

Identifica las actividades que le resultan de menor y mayor interés y dificultad, reconociendo y controlando sus reacciones frente a retos y obstáculos.

Articula saberes de diversos campos y establece relaciones entre ellos y su vida cotidiana. 
Tabla 2. Contenidos

\begin{tabular}{|c|c|c|}
\hline CONCEPTUAL & PROCEDIMENTAL & ACTITUDINAL \\
\hline 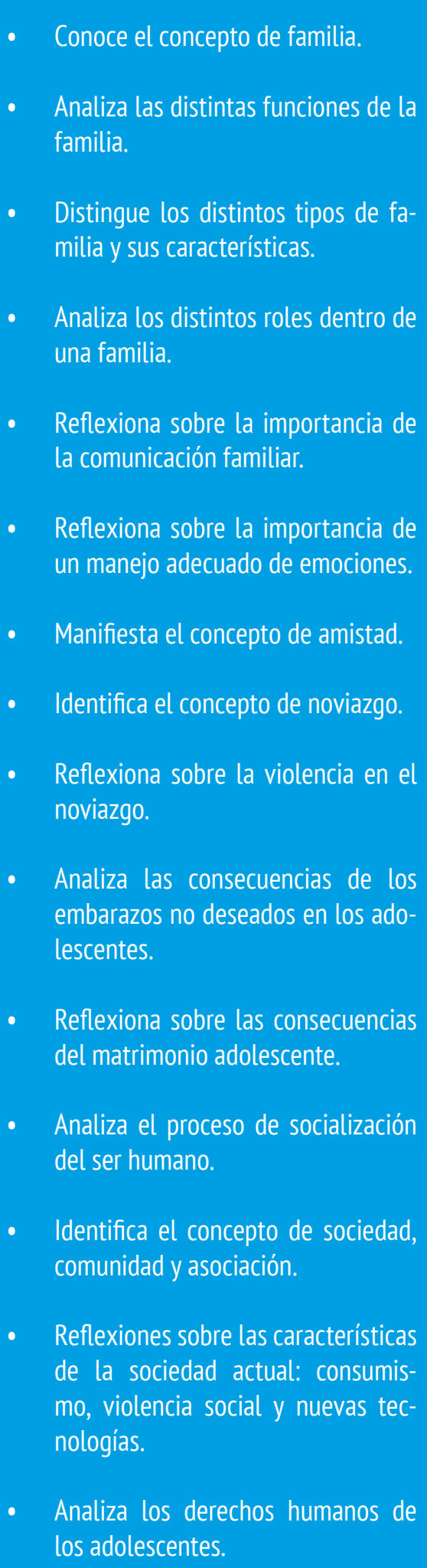 & 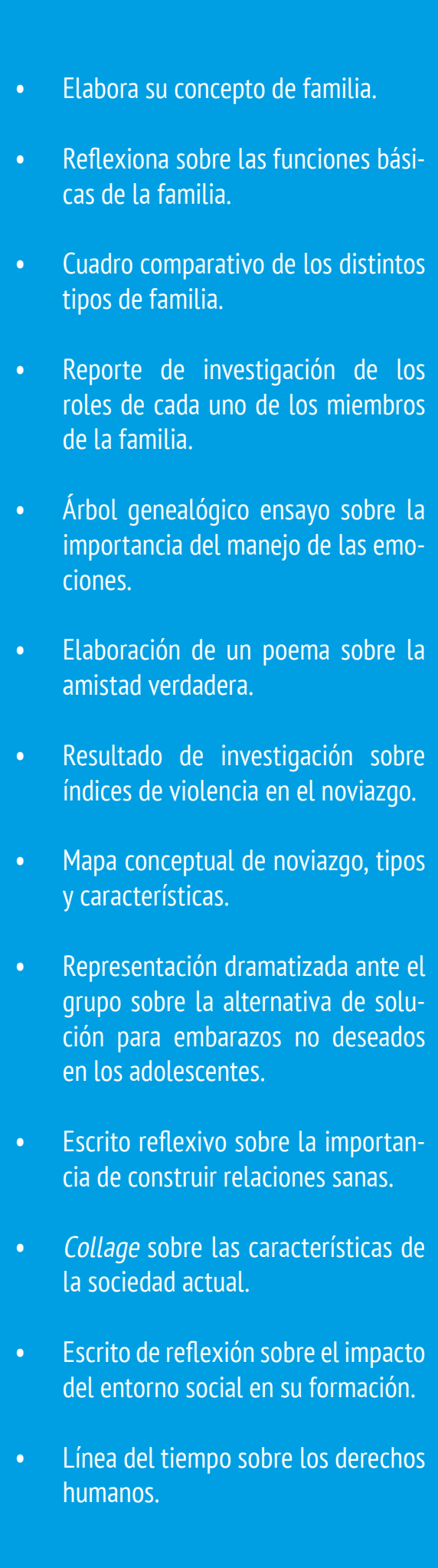 & $\begin{array}{l}\text { - } \\
\text { Practica el respeto por distintas for- } \\
\text { mas de vida. } \\
\text { guien responsable y único. } \\
\text { Colabora en las tareas encomenda- } \\
\text { das asumiendo un trabajo en equipo } \\
\text { con equidad. } \\
\text { Muestra la disposición para trabajar } \\
\text { en equipo de forma flexible, adap- } \\
\text { table, capaz de salir de esquemas } \\
\text { mentales rígidos. } \\
\text { Manifiesta actitudes positivas con } \\
\text { respecto a las relaciones humanas, } \\
\text { la honestidad, el respeto, la empatía } \\
\text { y la tolerancia. }\end{array}$ \\
\hline
\end{tabular}




\section{En este sentido, hemos de entender por dimensión el conjunto de potencialidades fundamentales con las cuales se articula el desarrollo integral de una persona.}

El propósito de estas actividades es que el alumno no se quede fuera de conocer temas y experiencias que le ayudarán a su proceso de maduración de forma holística, con el objetivo de desarrollar cada una de las dimensiones del ser humano. En este sentido, hemos de entender por dimensión el conjunto de potencialidades fundamentales con las cuales se articula el desarrollo integral de una persona; o también si se quiere, unidades fundamentales, de carácter abstracto, sobre las que se articula el desarrollo integral del ser humano. Estas dimensiones son la esfera ética, afectiva, espiritual, cognoscitiva y física.

\section{OBJETIVO GENERAL}

Establecer el m-learning como una herramienta de uso cotidiano dentro de la clase de Para escolares 3A, 3B, 3C, 4A y $4 \mathrm{BCCH}$.

\section{OBJETIVOS PARTICULARES}

- Analizar las temáticas de las TED Talks.

- Identificar las necesidades educativas del alumno.

- Identificar las necesidades socio afectivas del alumno.

- Sugerir estrategias de intervención de los ámbitos personales y sociales.

- Fomentar la formación académica por medios no convencionales.

- Desarrollar la participación crítica

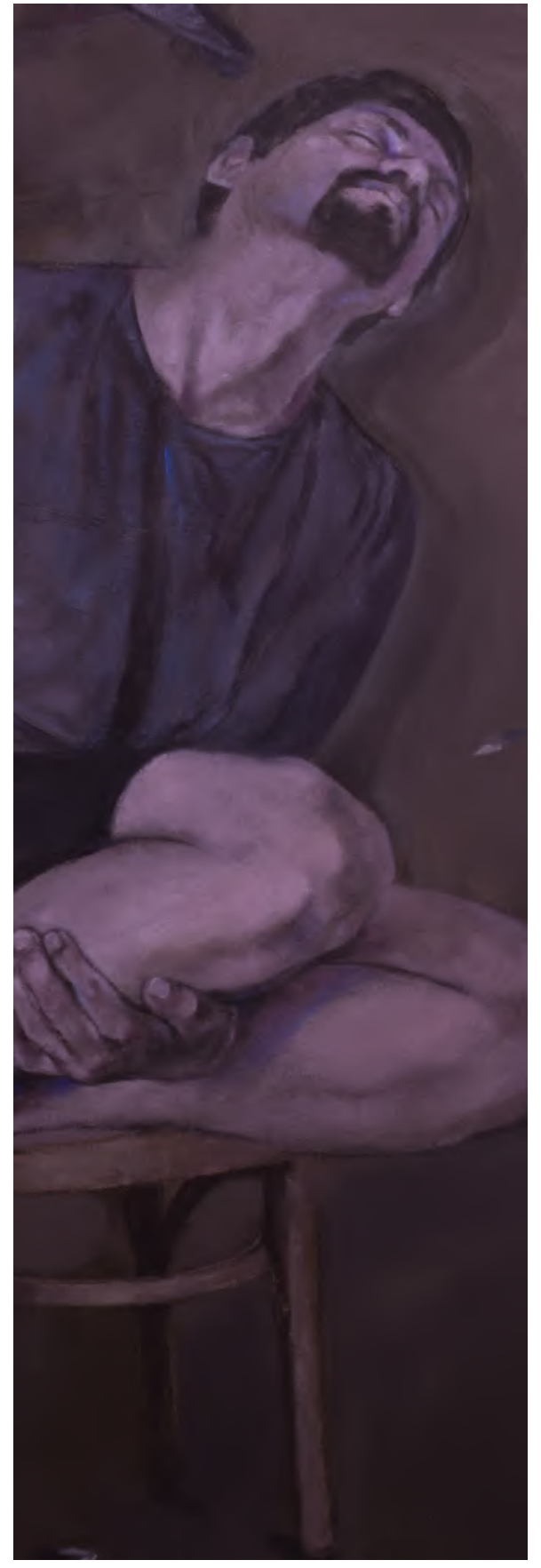

respetando con tolerancia la opinión de los demás.

\section{COMPETENCIAS DISCIPLINARES}

- Identifica el conocimiento social y humanista como una construcción en constante transformación.

- Valora todas las diferencias sociales, políticas, económicas, étnicas, culturales y de género y las desigualdades que inducen.

- Interpreta su realidad social a partir de los procesos históricos locales, nacionales e internacionales que la han configurado.

- Valora distintas prácticas sociales mediante el reconocimiento de sus significados dentro de un sistema cultural, con una actitud de respeto.

\section{ESTRATEGIA DIDÁCTICA O PROYECTO}

En la clase de Para escolares 3A, 3B, 3C, $4 \mathrm{~A}$ y $4 \mathrm{~B}$ dentro $\mathrm{CCH}$ se abordan temáticas que por su naturaleza la forma teórica no es opción que garantice la reflexión, cambio de conducta y/o motivación a mejorar los aspectos tratados. Estos aspectos giran en torno a la vida del adolescente que habían sido tratados de forma presencial, pero que en este proyecto da la opción de ser expuestos por medio de dispositivos móviles y el m-learning.

De esta forma el alumno puede dedicar 20 minutos de su día, que es la máxima 
Selección del Tema. El docente selecciona el tema a discutir de forma práctica una vez que se tenga abordada la teoría.

\section{Figura 1. Especificaciones}

Selección de la Conferencia. Dentro de la plataforma

TED (se puede descargar la app a dispositivos móviles)

se busca una o varias conferencias que refuercen el tema.
Debate. Por medio de Zoom se realiza un debate, siendo

el docente el moderador y los alumnos los participantes

para exponer sus reflexiones. duración de las pláticas para conocer el tema, en vez de las conferencias que pueden durar hasta 90 minutos, generando mayor atención al tema y sin distractores.

Las conferencias (TED Talks) permitirán cumplir con el apoyo de orientación educativa de la clase, para guiar y auxiliar al alumno a dar claridad en su entorno, dando un desarrollo personal, pues van dirigidas a promover la autoestima, las relaciones con sus pares y familia, a conocer y atender sus necesidades como persona, reconocer sus habilidades y talentos, así como sus errores y debilidades con el fin de mejorar como persona.

Identificado lo anterior el docente puede canalizar de ser necesario a los departamentos de tutorías, orientación educativa y psicopedagogía, comportamientos y expresiones que considere no sanas para intervenir en el desarrollo del alumno, esto por medio de lo que pueda observar en los debates por medio de ZOOM 0 de forma presencial. De esta forma se está trabajando un proyecto con una innovación didáctica con su formato de prácticas de intervención didáctica: la construcción de estrategias didácticas y medios para la enseñanza.

\section{ESPECIFICACIONES DEL PROYECTO}

Actividades y tareas: Retomando las especificaciones de la Figura 1, se trabajará de manera asincrónica y sincrónica con las siguientes actividades.

\section{RECURSOS HUMANOS}

Los docentes que conforman la academia de Para escolares 3 y 4 , guiados por el encargado del proyecto y para capacitar sobre el uso de dispositivos móviles en el aula de clase y sobre las aplicaciones a utilizar. En una primera instancia el encargado del proyecto puede dar los nombres de las conferencias y actividades a realizar y una vez que los maestros de la academia estén familiarizados con los conceptos del m-learning y las aplicaciones puede darse un trabajo colegiado para intercambio de información. Los alumnos que cursen el $3^{\circ}$ y $4^{\circ}$ semestre serán el público a quién va dirigido el proyecto durante su paso por el $\mathrm{CCH}$.

\section{RECURSOS FINANCIEROS}

Las APPS de TED y ZOOM no tienen costo, siendo un gran incentivo para aplicar el proyecto, pues no genera una carga económica para la institución la cual se ha visto afectada por los recortes presupuestales de la UJED por la crisis económica que atraviesa. La app de ZOOM no tiene costo los primeros 45 minutos, después de ello se necesita tener un plan mensual 0 anual de pago si se desea que las videollamadas (conferencias) tengan mayor duración, pero las clases del CCH tienen una duración de 45 a 60 minutos, así que se está trabajando en el tiempo adecuado

\section{COMPETENCIAS GENÉRICAS}

Son las que han de articular y dar identidad a la EMS y que constituyen el perfil del egresado del SNB son las que todos los bachilleres deben estar en capacidad de desempeñar; les permiten comprender el mundo e influir en él; les capacitan para continuar aprendiendo de forma autónoma a lo largo de sus vidas, y para desarrollar sus relaciones armónicas con quienes les rodean.

\section{COMPETENCIAS DISCIPLINARES}

Son las nociones que expresan conocimientos, habilidades y actitudes que consideran los mínimos necesarios de 


\section{Tabla 2.Actividades}

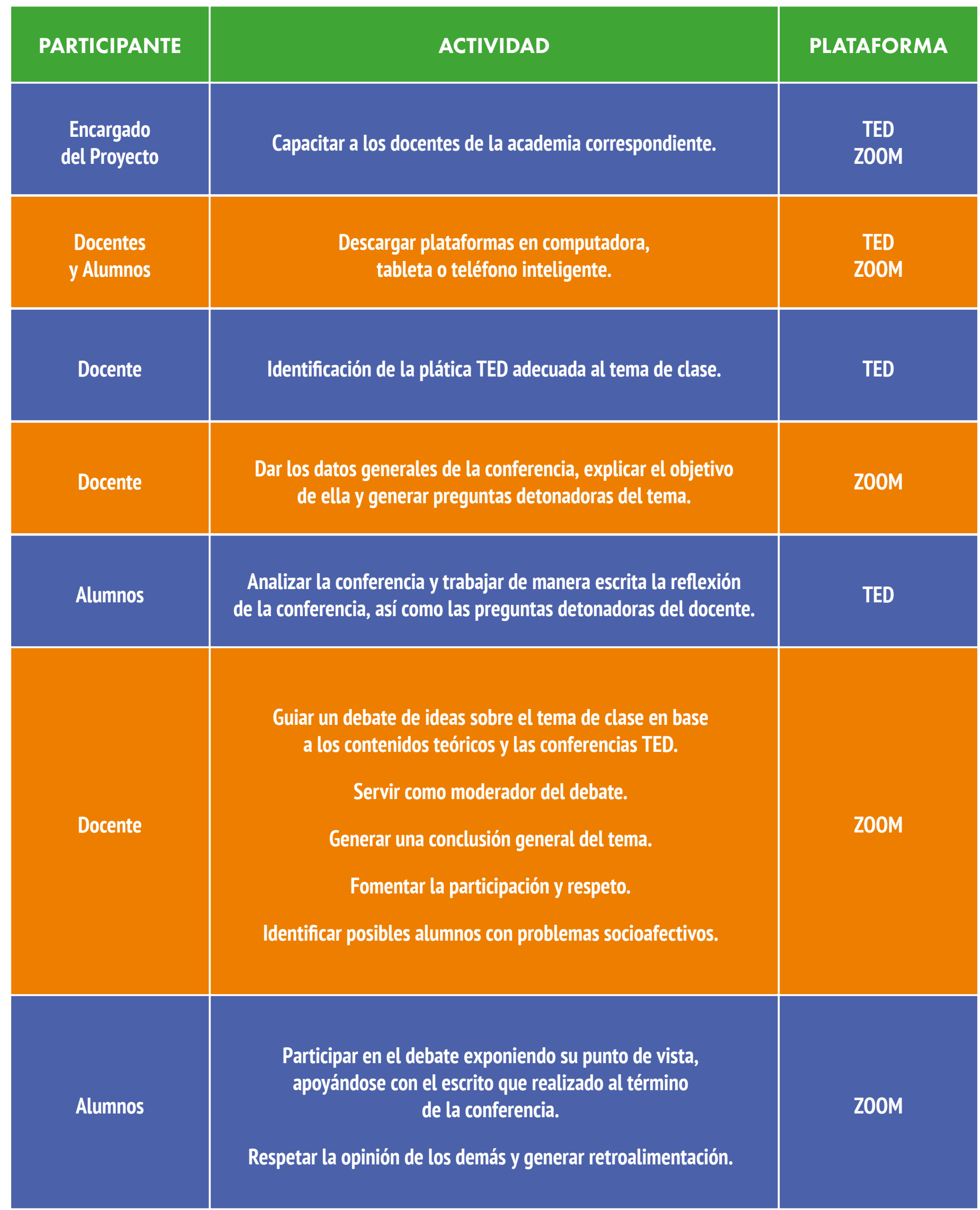


cada campo disciplinar para que los estudiantes se desarrollen de manera eficaz en diferentes contextos y situaciones a lo largo de la vida.

\section{E-TUTORING,}

\section{E-COACHING, E-MENTORING}

Se pueden ofrecer a los alumnos servicios que otorgan una dimensión humana o social de apoyo a lo largo del proceso de aprendizaje. El e-tutoring (tutoría en línea), e-coaching (preparación en línea) y e-mentoring (asesoramiento en línea) ofrecen apoyo individual y retroalimentación a los alumnos a través de herramientas y técnicas de facilitación.

\section{E-LEARNING SINCRÓNICO $Y$ ASINCRÓNICO}

En e-learning se usan los términos síncrona y asíncrona para referirse a las dos posibilidades de interacción o comunicación entre profesor y alumno.

Asíncrona. La comunicación asíncrona permite que el profesor y el alumno interactúen en lugares diferentes y en tiempos distintos. No existe una comunicación directa 0 en tiempo real entre profesor y alumno. Las herramientas de comunicación 0 interacción más utilizadas para esta modalidad de aprendizaje son: correo electrónico, listas de correo, foros de discusión, wikis, blog.

Síncrona. La comunicación síncrona es una modalidad de aprendizaje en que el profesor y el alumno se escuchan, se leen $y / 0$ se ven en el mismo momento, independientemente de que se encuentren en espacios físicos diferentes. Esto permite que la interacción se realice en tiempo real como ocurre en las clases presenciales. Esta modalidad se desarrolla con herramientas como: el chat, pizarra electrónica, audioconferencia 0 videoconferencia.
Metodología. En la presente ponencia se encuentra la metodología cuantitativa, así las técnicas de investigación de acción, debido a que se presenta una estrategia para mejorar el aprendizaje en los alumnos, pero también apoyadas con el método documental, pues se recopiló datos sobre las teorías que sustentan el empleo de recursos lúdico.

\section{RESULTADOS}

Al emplear las conferencias TED y las reuniones ZOOM, los alumnos se muestran motivados por la clase perdiendo el miedo por participar de forma verbal o de forma escrita frente a sus compañeros, ya que se crea un ambiente de confianza donde los compañeros muestran respeto por las opiniones y participaciones de los demás. Entendieron temas que van más allá de la teoría pues por medio de las pláticas de personas que son famosas,

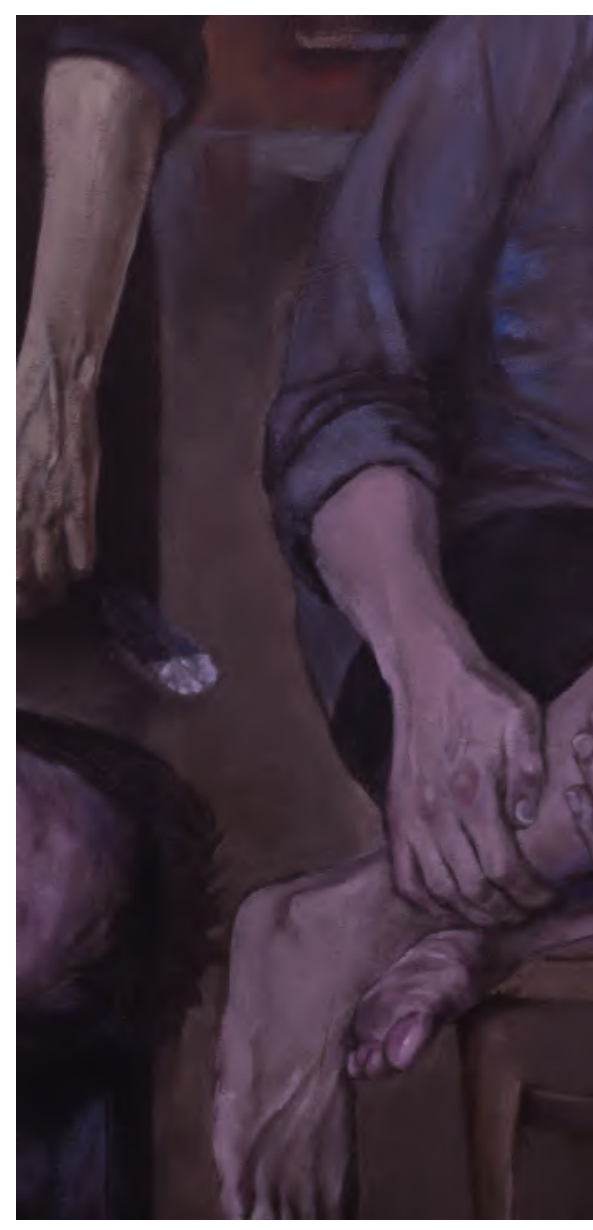

109 jóvenes con quienes se identifican, vocabulario que conocen y el breve tiempo en el que se explica el tema les pareció interesante y los motivó a la reflexión.

La participación en clase aumentó y entre ellos mismos hacían investigación de las pláticas TED con base a los temas a trabajar, les gustaba el intercambio de ideas y las pláticas que proponían. La presentación de los trabajos pasó de ser un corta y pega de internet con conceptos generales a una reflexión más profunda del tema.

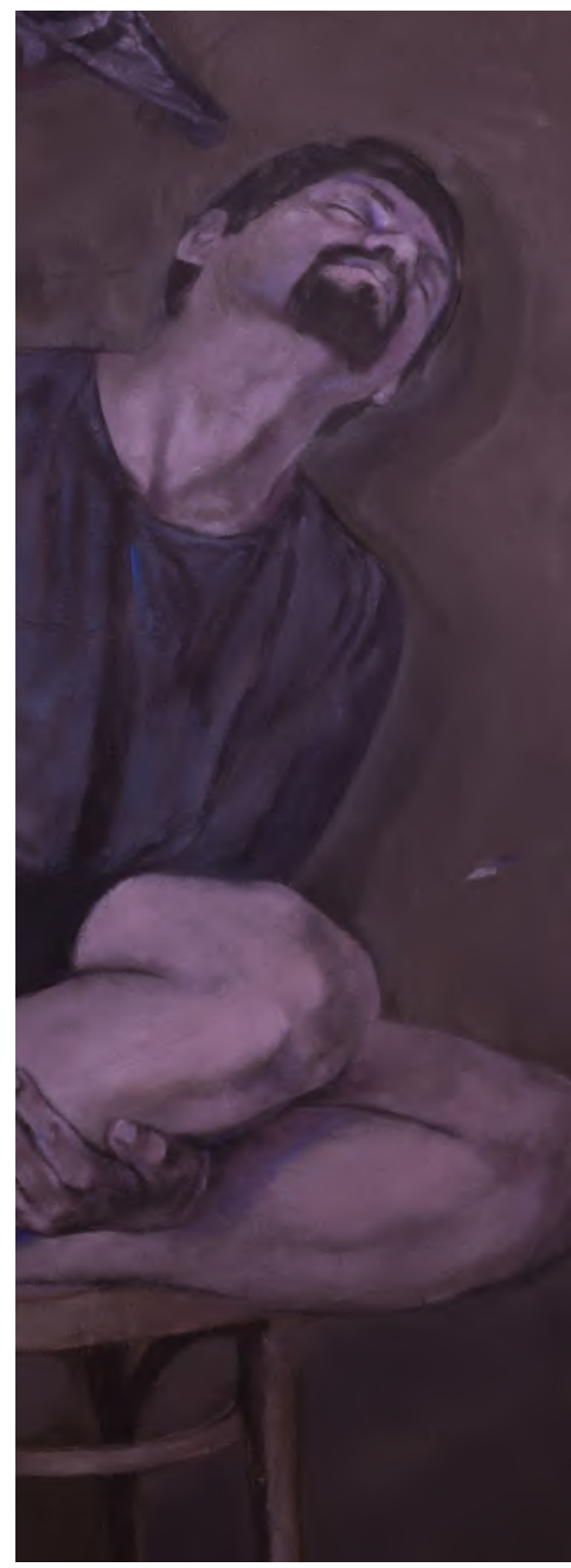

Preparatoria 8 UANL 


\section{REFERENCIAS}

Cárdenas, T. (2013). REDIE. Obtenido de https://redie.mx/librosyrevistas/libros/ como_elaborar_proyectos_de_innovacion.pdf

México, G. d. (2020). Covid 19. Obtenido de https://coronavirus.gob.mx/covid-19/

Olmedo, E. M. (2020). UAEH docencia. Obtenido de https://www.uaeh.edu.mx/docencia/VI Lectura/maestria/documentos/LECT35.pdf

Secretaria de Educación Pública. Obtenido de https://www.dgb.sep.gob.mx/informacion-academica/mapas curriculares.php

TED. (2020). TED. Obtenido de https://www.ted.com/about/our-organization FAO (2014). Metodología del e-learning.

Grau-Perejoan, 0., (2008). Formación on line. Educación Médica, 11(3), 139-146. Job-Wizards. (2020). Job-Wizards. 


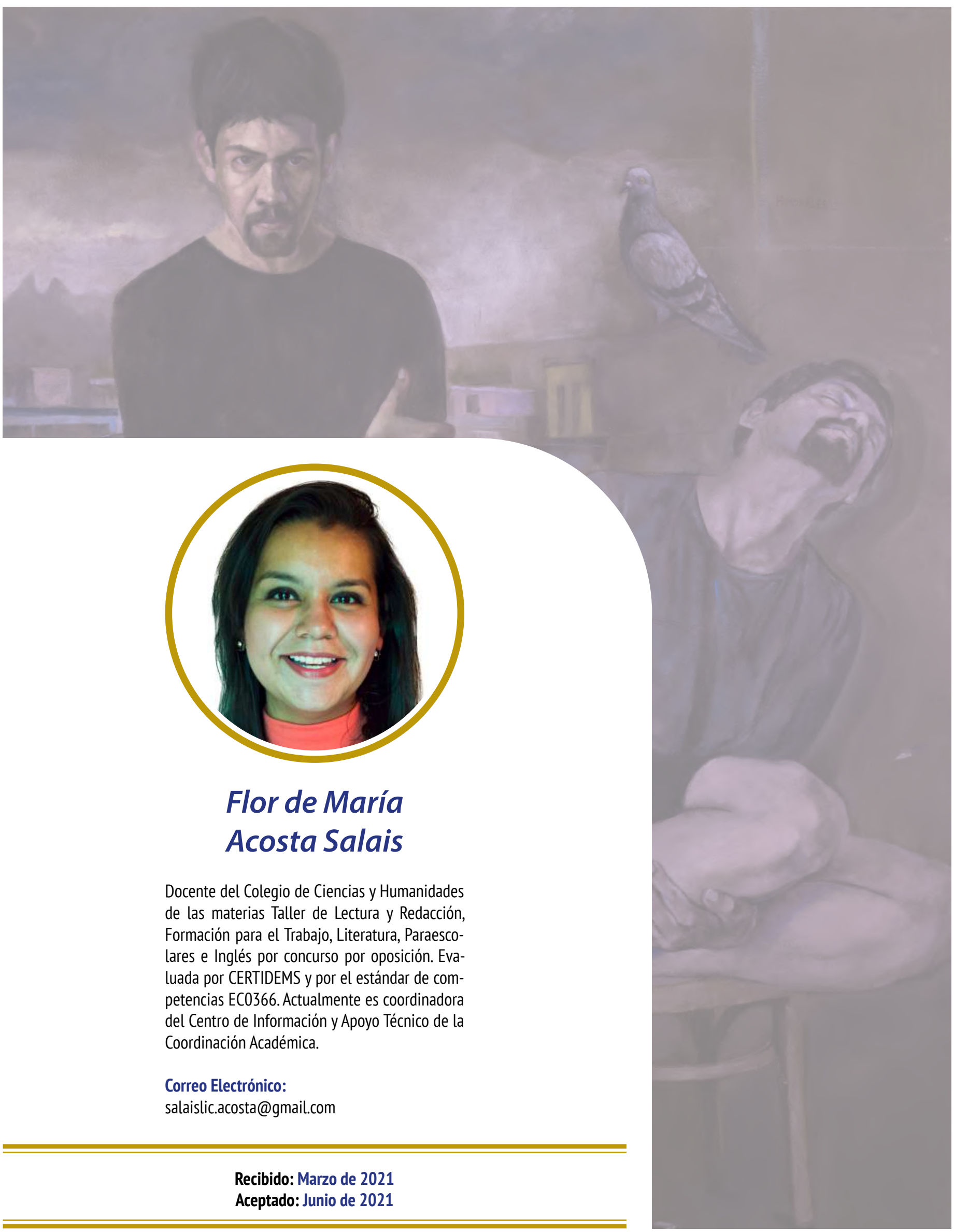




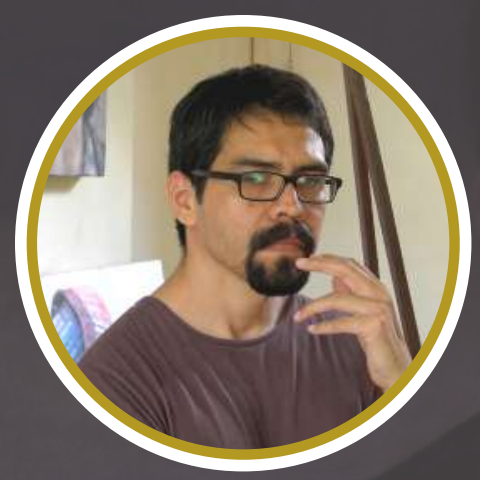

\section{Héctor Manuel Morales Orona}

Egresado de la Facultad de Artes Visuales de la Universidad Autónoma de Nuevo León. Inicia su profesión en el área de las artes gráficas, ilustrando en publicaciones de casas editoriales. Al mismo tiempo alterna esta actividad con la pintura mural. Después de este período inicial, su inquietud creativa se dirige al teatro, donde realiza estudios de arte dramático, cuyo aprendizaje originó nuevas reflexiones sobre el concepto de representación, y la experiencia del juego.

Más adelante, se dedica al estudio de las técnicas antiguas de la pintura haciéndolas su especialidad, y diversifica su profesión como pintor-dibujante y profesor de artes. Desde las técnicas antiguas y la importancia de los materiales, ha encontrado un campo nuevo de resignificaciones conceptuales, representando estas ideas sobre la materia, una fuerte línea de investigación que actualmente trabaja.

Cuenta con múltiples exposiciones colectivas e individuales. Ha participado en ediciones de la Reseña de la Plástica de Nuevo León y su obra se ha expuesto en México, Italia, Cuba y Canadá.

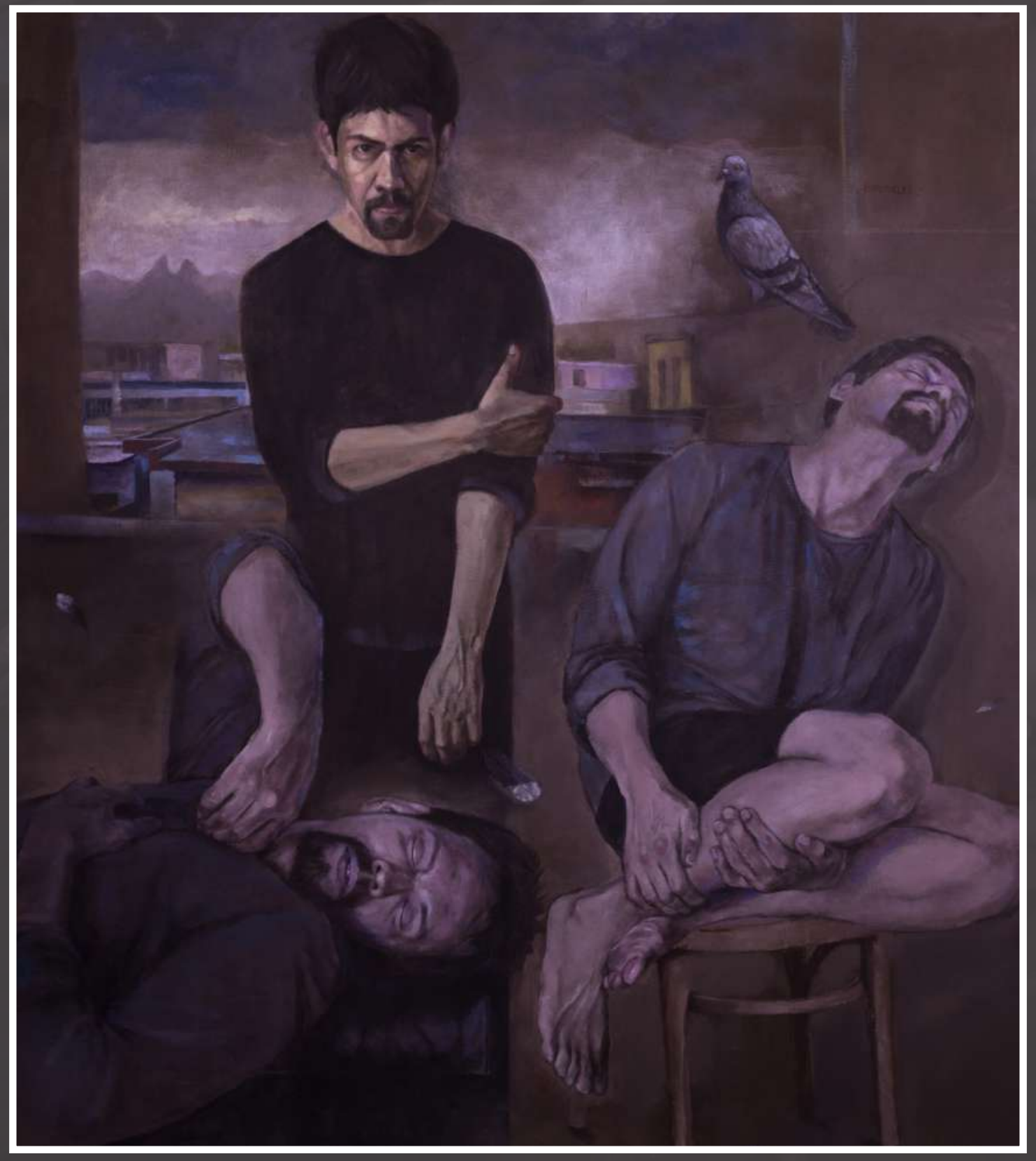

